\title{
Design of a Mobile Augmented Reality-based Indoor Navigation System
}

\author{
Xin Hui Ng, Woan Ning Lim \\ Research Centre for Human-Machine Collaboration \\ Department of Computing and Information Systems \\ School of Science and Technology \\ Sunway University, Malaysia \\ ( xinhui1216@gmail.com, woanningl@ sunway.edu.my )
}

\begin{abstract}
GPS-based navigation technology has been widely used in most of the commercial navigation applications nowadays. However, its usage in indoor navigation is not as effective as when it is used at outdoor environment. Much research and developments of indoor navigation technology involve additional hardware installation which usually incur high setup cost. In this paper, research and comparisons were done to determine the appropriate techniques of indoor positioning, pathfinding, and route guidance for an indoor navigation method. The aim of this project is to present a simple and costeffective indoor navigation system. The proposed system uses the existing built-in sensors embedded in most of the mobile devices to detect the user location, integrates with AR technology to provide user an immersive navigation experience. In this project, an indoor navigation mobile application was developed and tested. The development demonstrates the usage of IndoorAtlas which enables indoor positioning through technology fusion to detect user's position and obtain the route to destination, and ARCore to display AR guidance using the computed route. Surveys were carried out to gauge the efficiency of the method and to gather the feedback from the participants. The architecture of the method and the demonstration of the application is presented.
\end{abstract} reality;

Keywords-indoor navigation; indoor positioning; augmented

\section{INTRODUCTION}

GPS-based outdoor navigation system on smartphones has been widely used in recent years because of its high level of precision. However, indoor navigation is not as common as outdoor navigation due to the incompatibility of GPS signals inside complex multi-floor buildings. As a result, unlike outdoor navigation, there are relatively lesser development on commercial indoor navigation systems.

This paper proposes an efficient and cost-effective method by using the built-in sensors of mobile devices and the augmented reality (AR) technology to provide real-time indoor navigation. This method does not require installation of additional hardware, the user's current position is detected by using the mobile devices' magnetic field, Wi-Fi signals and inertial sensor and the route to destination is calculated based on ACO algorithm to provide navigation guidance. AR technology is used to replace the traditional map to provide an immersive virtual navigation direction. A mobile application prototype was developed in this project to provide navigation within Sunway University campus.

\section{RELATED WORK}

An indoor navigation system is comprised of 3 important components which are positioning, wayfinding, and route guidance. Positioning refers to determining user's current position, while wayfinding focuses on searching the route from user's position to a specific destination. Route guidance is the navigation directions illustrating the route.

\section{A. Indoor Positioning Techniques}

In order to determine which positioning technique is more suitable, the popular techniques, including GPS, Wi-Fi-based positioning, BLE beacon-based positioning, magnetic fieldbased positioning, and vision-based positioning, are discussed.

GPS signals can be weakened when there are obstacles, such as the walls and roofs, between the direct path of the receivers and the satellite [1]. Besides, it is also extremely difficult to determine the floor level [2]. Therefore, GPS is too weak to provide usable positioning information indoors, especially in multi-floor buildings.

Wi-Fi-based positioning has low hardware installation cost and high availability because Wi-Fi are commonly deployed inside buildings nowadays [3]. However, as it uses fingerprinting technique to collect $\mathrm{Wi}-\mathrm{Fi}$ signals and associate the signals with location, the process is time-consuming. The obstacles inside buildings might also cause multipath effects which leads to unreliable accuracy [4].

BLE beacon consumes less power and the beacon is small in size, cheap and easy to set up [5]. Besides, in terms of power consumption, BLE is more energy efficient than other wireless signals [6]. However, similar to Wi-Fi-based positioning, the fingerprinting process is time-consuming, and BLE positioning also has the risk of multipath effects and even more serious than Wi-Fi-based positioning [5].

Magnetic field signals are natural resources, hence, no additional infrastructure is needed. It also will not be affected by obstacles, such as walls or roofs [7]. However, metal element might cause hard-iron and soft-iron effect, which 
affects its accuracy [8]. The fingerprinting process is also time consuming.

Vision-based positioning can be categorized into markerless tracking and visible marker-based tracking [9]. Markerless vision recognition uses natural features, such as corners and textures, of surrounding environment to provide positioning information [10], therefore, no additional infrastructure is needed. However, high computational and memory resources are required because the system will have to keep scanning and comparing the environment with large-scale database [11]. This technique is also unreliable if used in dynamic changing environment [7]. For example, when the table or chair is moved, the system might not recognize the location anymore. So, we will need to update the database frequently to match the environment, which is costly.

Visible marker refers to any image-based code, such as QR code [12]. The marker will be placed at certain position to provide location information when it is scanned. By using this technique, additional cost is required to place the markers around the building. Other than that, in order to update user's position, the user will have to keep finding and scanning the marker. Both methods of vision-based positioning can be relatively accurate for tracking objects in spaces with small dimensions, which is suitable for indoor positioning [13]. However, both will be affected by sources, such as light and weather [7]. For example, when there is a black out, both techniques will not work.

As mentioned, GPS signals alone is too weak to provide indoor positioning. Wi-Fi and BLE beacon signals might be affected by obstacles inside the building, vision-based positioning will also be affected by light sources. Therefore, the best solution would be a technology fusion positioning system, which combines different technologies to complement each other. Based on [14], the fusion technique of combining $\mathrm{Wi}-\mathrm{Fi}$ and magnetic field signals provides median accuracy of 1.20 meters, which is better than solely Wi-Fi. Furthermore, the technique proposed in [15] with the integration of Wi-Fi and magnetic field can reliably achieve 0.836 meters of accuracy in the experimental environment. Also, as most buildings already have Wi-Fi installed and magnetic field is natural resource, additional infrastructure cost can be minimized. However, the technique is time-consuming because it requires fingerprinting. The comparisons of the indoor positioning techniques are summarized in TABLE I.

\section{B. Pathfinding Techniques}

The popular pathfinding techniques include Dijkstra's algorithm, A* algorithm and ACO algorithm [18]. Dijkstra's algorithm is a mature shortest pathfinding algorithm between two nodes, while $A^{*}$ algorithm is an extension of Dijkstra's algorithm that combines the features of uniform-cost search and pure heuristic search to search for optimal path [19]. On the other hand, Ant Colony Optimization (ACO) algorithm mimics the behaviour of ants walking around the graph. As ants will follow the trail with more pheromones, ACO algorithm also use the same approach to compute the path [16].

According to [18], $\mathrm{A} *$ algorithm is more efficient than Dijkstra's algorithm. Nevertheless, as shown in the testing results in [16], ACO algorithm has higher accuracy and less time cost in searching route as compared to $A^{*}$ algorithm. In the context of indoor environment, the computation cost of $\mathrm{A}^{*}$ algorithm will be much higher than ACO algorithm. In few cases, the path searched using $\mathrm{A}^{*}$ algorithm is a bit shorter than using ACO algorithm, however, the time spent using $\mathrm{A}^{*}$ algorithm is 8 times more than using ACO algorithm. Therefore, [16] indicates that ACO algorithm is suitable for pathfinding in indoor environment.

TABLE I. COMPARISONS OF INDOOR POSITIONING TECHNIQUES

\begin{tabular}{|c|c|c|c|}
\hline \multirow[b]{2}{*}{$\begin{array}{l}\text { Positioning } \\
\text { Technology }\end{array}$} & \multicolumn{3}{|c|}{ Feature } \\
\hline & $\begin{array}{c}\text { Indoor } \\
\text { Positioning } \\
\text { Accuracy } \\
\text { Range } \\
\end{array}$ & $\begin{array}{c}\text { Cost } \\
\text { Effectiveness }\end{array}$ & Robustness \\
\hline GPS & $>40 \mathrm{~m}$ & Low cost & $\begin{array}{l}\text { - Weak signal strength } \\
\text { indoors }\end{array}$ \\
\hline Wi-Fi & $\begin{array}{l}30-40 \mathrm{~m} \\
\quad[16]\end{array}$ & Low cost & $\begin{array}{l}\text { - Multipath effect } \\
\text { - Time consuming for } \\
\text { fingerprinting }\end{array}$ \\
\hline BLE Beacon & $\begin{array}{c}10-20 \mathrm{~m} \\
{[16]}\end{array}$ & High cost & $\begin{array}{l}\text { - Multipath effect } \\
\text { - Time consuming for } \\
\text { fingerprinting }\end{array}$ \\
\hline Magnetic Field & $2-6 m[17]$ & Low cost & $\begin{array}{l}\text { - Metal elements } \\
\text { interference } \\
\text { - Time consuming for } \\
\text { fingerprinting }\end{array}$ \\
\hline Visible Marker & Unreliable & High cost & $\begin{array}{l}\text { - Line of sight requirement } \\
\text { - Light source interference }\end{array}$ \\
\hline $\begin{array}{l}\text { Markerless } \\
\text { Vision } \\
\text { Recognition }\end{array}$ & Unreliable & Low cost & $\begin{array}{l}\text { - Light source interference } \\
\text { - High computational and } \\
\text { memory resources } \\
\text { requirement }\end{array}$ \\
\hline $\begin{array}{l}\text { Fusion (Wi-Fi + } \\
\text { Magnetic Field) }\end{array}$ & $1.2 \mathrm{~m}$ & Low cost & $\begin{array}{l}\text { - Time consuming for } \\
\text { fingerprinting }\end{array}$ \\
\hline
\end{tabular}

\section{Route Guidance Techniques}

Digital map is commonly used to provide indoor guidance. However, research proved that computer graphic is not sufficient to represent the real world, so, it is difficult for users to match the map with real world. It also causes confusion because users will need to compare the actual landmark and direction with those in the map [20].

In order to enhance user experience, AR has arisen to provide more immersive guidance. AR is a technology which augment the real-world camera view with virtual images. Unlike digital map which provides a third-person view of the world, AR shows a first-person view of target direction which allows users to interact with the device naturally [21]. AR is also proven to reduce the time and recognition workload of human wayfinding behaviors [22]. 


\section{SYSTEM DESIGN}

This section presents the system's architectural design and user interface.

\section{A. Architectural Design}

The major components of the system and the interactions are shown in Fig. 1.

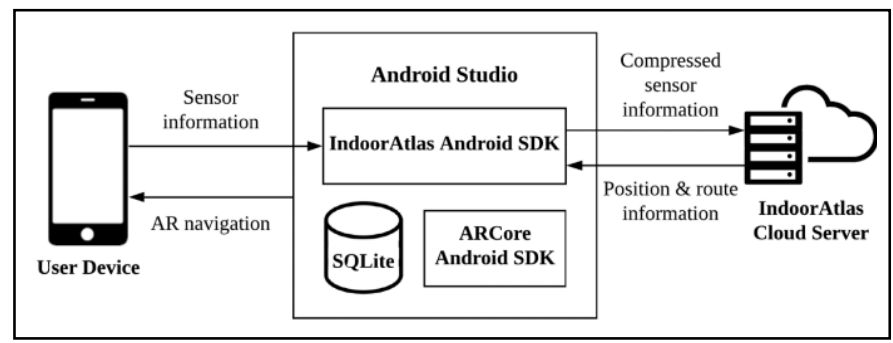

Fig. 1. Architecture Diagram

The AR indoor navigation mobile application is developed using Android Studio with the integration of IndoorAtlas Android SDK [23] and ARCore Android SDK [24]. SQLite local database is used to store the venues information.

IndoorAtlas SDK uses magnetic field, Wi-Fi signals and inertial sensor to provide indoor positioning and pathfinding services, while ARCore SDK is used to provide AR guidance on top of real-world camera view.

When the user starts the navigation, the sensor information in the mobile device will be gathered and sent to the IndoorAtlas Cloud server. The position and wayfinding information will be computed and feedback to the devices. After getting the position and route, AR navigation guide will be displayed to guide the user to the destination.

As discussed in previous section, sensor fusion positioning technology has better accuracy than the technology that only uses 1 type of sensor source. By fusing different sensor sources, including magnetic field, Wi-Fi signals and inertial sensor, IndoorAtlas provides last meter accuracy indoor positioning [23].

\section{B. User Interface Design}

Fig. 2 presents the user interface and navigation graph of the AR indoor navigation mobile application. The user can select their destination by navigating through the categories and sub-categories of venue. Once selected, the route will be calculated and AR navigation screen will be shown with an automated 3D robot leading the user to their destination.

\section{IMPLEMENTATION}

As the solution was developed for the navigation within Sunway University campus, the venues information and floor plans of the university were gathered.

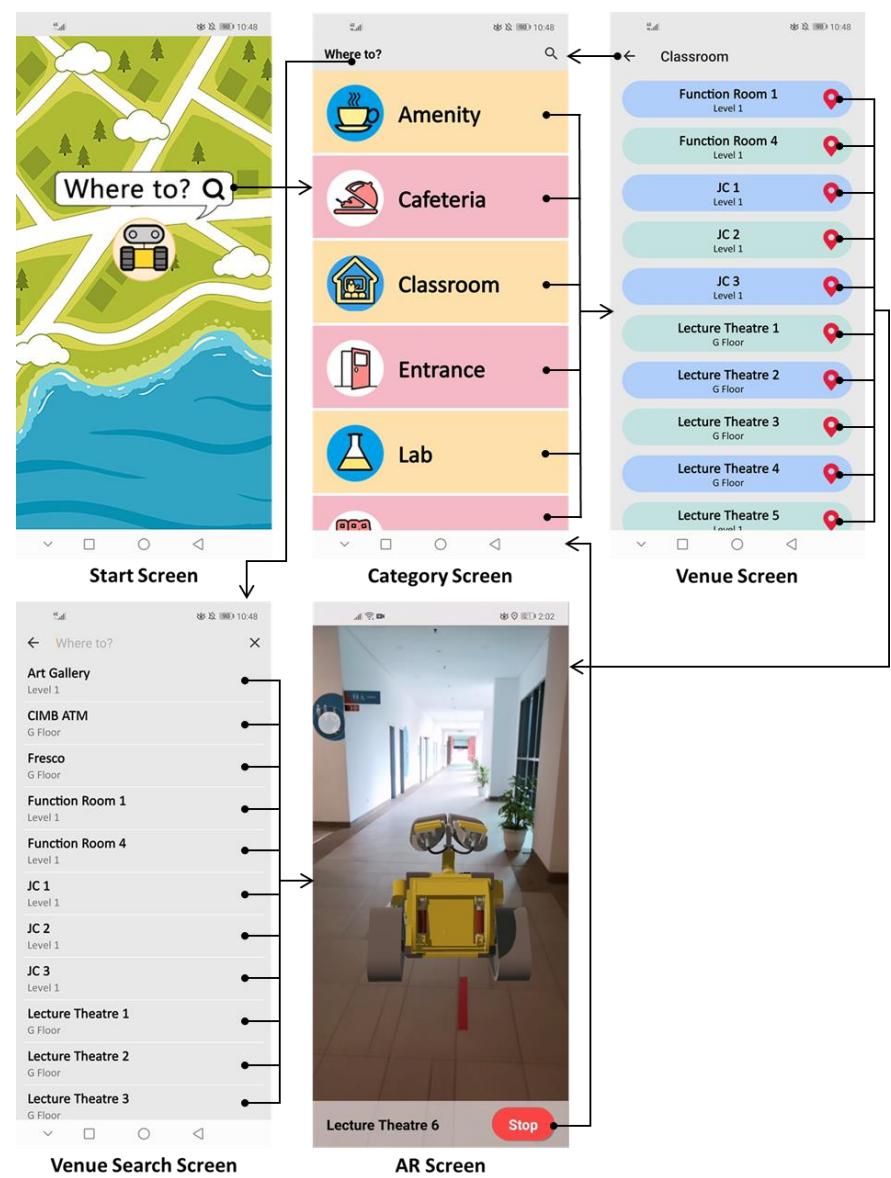

Fig. 2. User Interface and Navigation Graph

\section{A. Platform}

The AR indoor navigation system was implemented in the form of mobile application using Android Studio in Java, hence, the application is available on Android platform. IndoorAtlas SDK was used to get user's current position and the route information and ARCore SDK was used to display the route as AR images to guide the user. As the application only requires storing the venue information, a simple and lightweight local SQLite database was adopted.

\section{B. Activities}

At the beginning of the implementation, a mobile application with venues menu and searching function was developed using Android Studio based on the prototype design. The database was also implemented to store Sunway University's venue details, such as the name, venue category and floor level.

Then, to enable indoor positioning using IndoorAtlas SDK, the venues, location information and floor plans of Sunway University were uploaded to IndoorAtlas web application.

After uploading the floor plans, the points of interest, such as the cafeterias, library and classrooms, were added on top of the floor plans as shown in Fig. 3. By adding the points of interest, the application will know the location of each venue on the campus. 


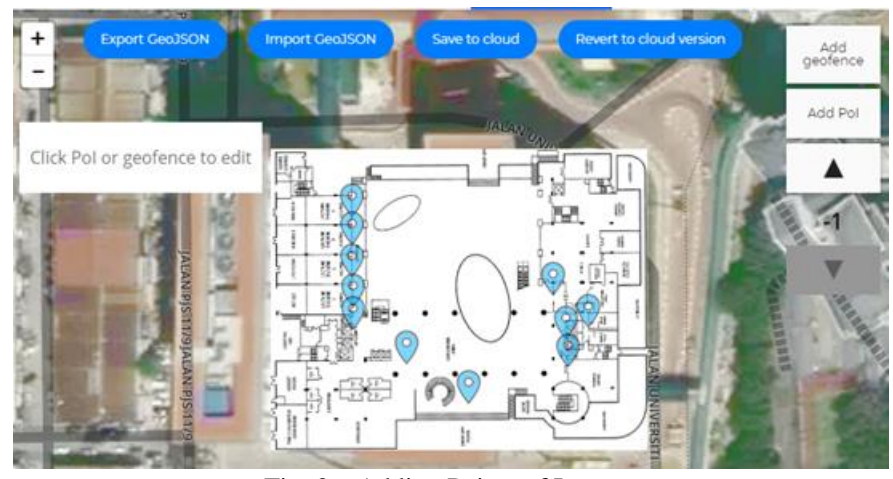

Fig. 3. Adding Points of Interest

Then, in order to get the route from a point to another, the wayfinding graph with nodes and edges was added to the floor plans as shown in Fig. 4.

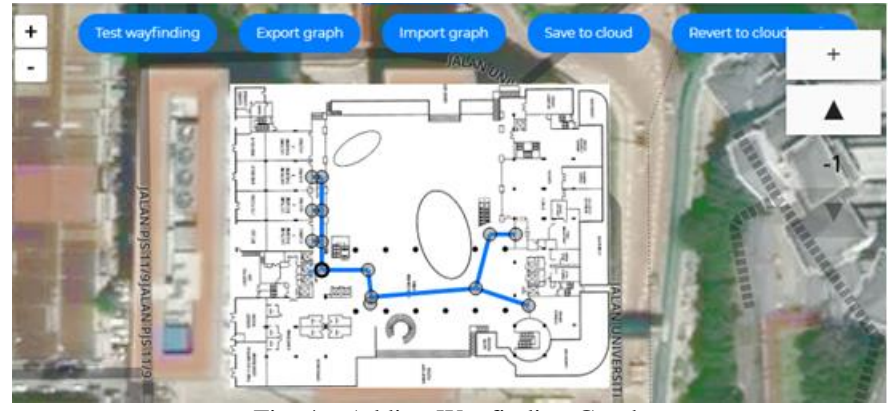

Fig. 4. Adding Wayfinding Graph

After uploading the floor plans and wayfinding information to the, fingerprinting had been carried out by using MapCreator 2 as shown in Fig. 5. The fingerprinting process involves calibrating the device's sensors and physically walking around Sunway University campus to collect the location information, such as magnetic field and Wi-Fi signals, inside the building.

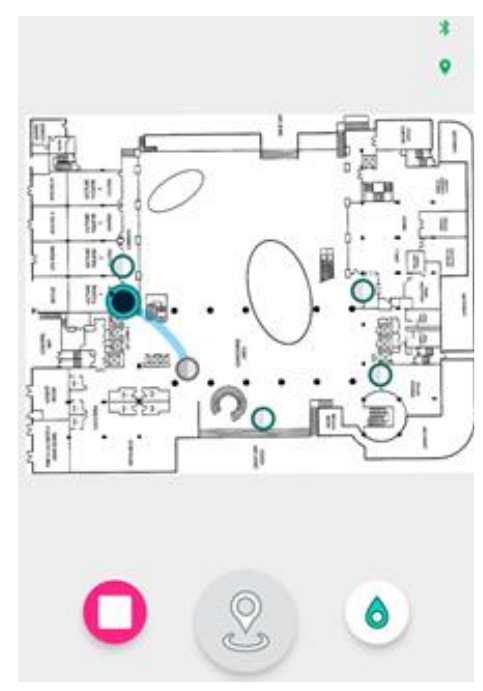

Fig. 5. Fingerprinting
The data gathered from the fingerprinting process is stored in the cloud and the signals map is generated for user's position computation.

When a venue is selected as the destination in the mobile application, the user's position will be detected to obtain the route to the destination. Once the route is successfully computed, the AR robot guide will then be presented on top of the camera view and ask the user to follow its navigation. The robot guide will then move, and turn based on the route to guide the user to the destination. By computing the difference of the current heading of the device and the heading of the next node, the robot guide can be turned and moved to the next node of the route. Once arrived at the destination, a dialog will be prompted to inform the user.

Fig. 6 presents the screenshots of the AR navigation process. The video demonstrations can be found at https://bit.ly/3hCrvLW.
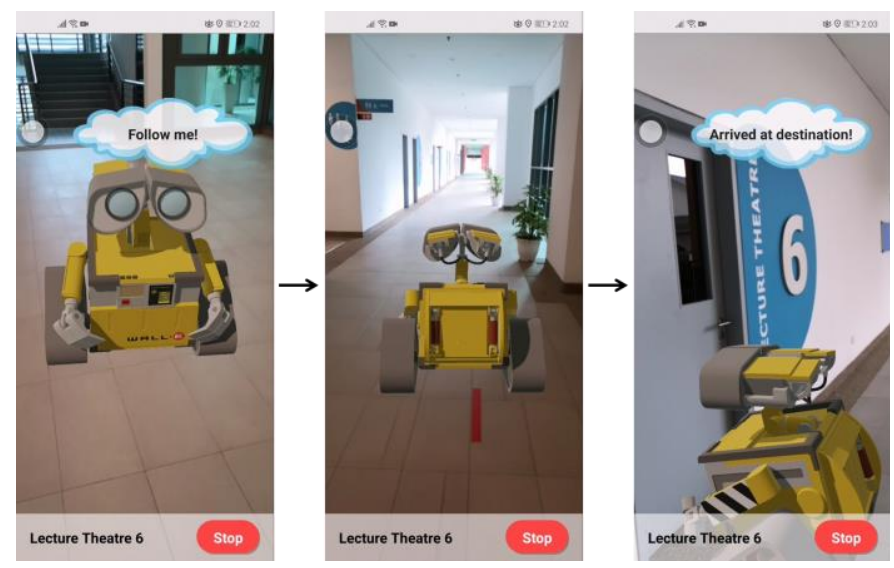

Fig. 6. AR Navigation

\section{RESUlTS AND DisCUSSIONS}

A survey has been carried out to gather user's opinions towards the mobile application. As the application is developed for Sunway University campus navigation, the target population of this survey is the students who are studying at Sunway University. 10 students had participated in the survey. The first question of the questionnaire is a demographic question to understand the familiarity of respondents against the Sunway Campus. 7 out of 10 respondents have been studying at Sunway University for more than 7 months. The other 2 respondents have been studying at the university for less than 12 months and 1 respondent for 7 to 12 months

\section{A. Survey}

The respondents were asked to use the mobile application on the provided smartphone to navigate themselves from their current location to Sunway Campus Library (Main Entrance) located at Ground Floor of the university. After using the application, they were asked to fill in the questionnaire as shown in TABLE II. 


\begin{tabular}{ll}
\hline Q1 & $\begin{array}{l}\text { Do you have difficulty finding your way to your destination at } \\
\text { Sunway University without any navigation assistance? }\end{array}$ \\
\hline Q2 & I could easily find the way to my destination by using the app. \\
\hline Q3 & I could clearly understand the directions shown on the app. \\
\hline Q4 & The app is helpful in guiding me to my destination. \\
\hline Q5 & What improvements do you think can be made to the app? \\
\hline Q6 & Will you use this app in the future when needed? \\
\hline
\end{tabular}

\section{B. Survey Responses}

Question 1 aims to investigate whether the participants find it difficult to reach their destinations at Sunway University without using the navigation application. All of the respondents find it difficult to find their way in the campus.

Question 2 to Question 4 allow the participants to rate the mobile application with four-point Likert scale, with 1 as 'Strongly Disagree' and 4 as 'Strongly Agree'. The responses are summarized in Fig. 7.

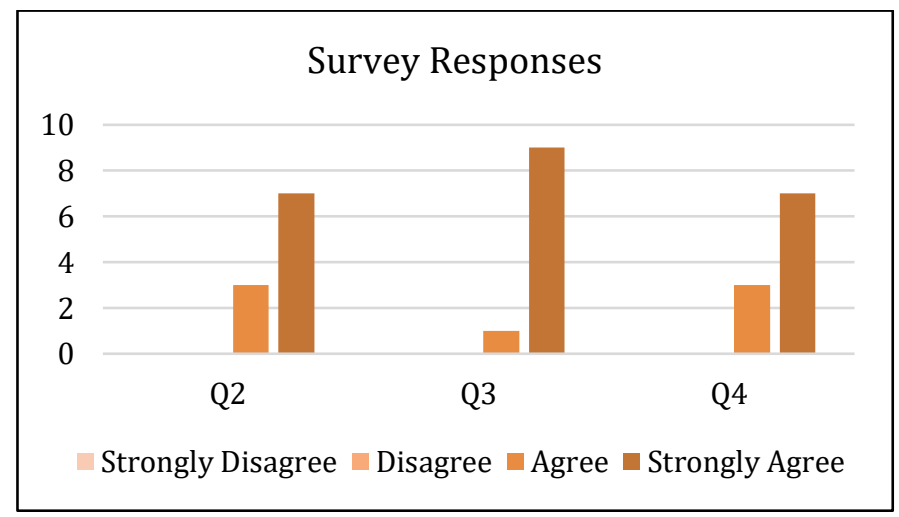

Fig. 7. Survey Responses

Generally, the participants responded positively towards the developed solution. All respondents agreed with the statements, which indicates that they could clearly understand the directions shown on the application and think that the application is helpful in guiding them to their destinations. Besides, the results of Question 6 show that all the participants were positive in using the application for their future navigation needs.

However, based on the responses of Question 5 there are some improvements suggested by the respondents that can be made to the application. Question 5 is not compulsory for the respondents to answer, hence there are only 6 responses collected from the participants. Amongst the suggestions are, instead of moving the AR robot guide straight to the destination, the application should be modified to make the AR robot stop and wait for the user at each node position. When the user reaches the position of the AR robot, then only the route be computed and move the AR robot to the next node. The participant also suggested that more venues at the university should be covered

\section{CONCLUSION AND FUTURE WORKS}

In conclusion, the indoor navigation system using the mobile devices built-in sensors and AR technology able to provide users an immersive navigation experience. It successfully detected user's position and guided the user to the destination by displaying AR navigation directions on top of the real-world camera view.

The application was tested in Sunway University campus with positive feedbacks from the survey participants. Generally, all the participants responded that the application was helpful to them and that they would use the application in the future. However, as suggested by the respondents, there are room for improvements in the AR robot navigation design.

This prototype is designed for same floor level navigation only, future works can be done to expand it to multi-floor navigation. The multi-floor positioning and wayfinding have already been implemented in the application; hence the multifloor navigation could be easily achieved by modifying the AR robot design.

Finally, as this project aims to provide a simple and costeffective AR navigation service for indoor environment, the proposed solution could be implemented in many contexts other than university campus, such as shopping malls, airports, and hospitals.

\section{REFERENCES}

[1] M. B. Kjærgaard, H. Blunck, T. Godsk, T. Toftkjær, D. L. Christensen, and K. Grønbæk, "Indoor Positioning Using GPS Revisited," in Pervasive 2010, 2010, pp. 38-56.

[2] D. Macias-Valadez, R. Santerre, S. Larochelle, and R. Landry, "Improving vertical GPS precision with a GPS-over-fiber architecture and real-time relative delay calibration," GPS Solutions, vol. 16, no. 4, pp. 449-462, 2012.

[3] S. He and S. H. G. Chan, "Wi-Fi fingerprint-based indoor positioning Recent advances and comparisons," IEEE Communications Surveys and Tutorials, vol. 18, no. 1, pp. 466-490, 2016.

[4] C. Yang and H. R. Shao, "WiFi-based indoor positioning," IEEE Communications Magazine, vol. 53, no. 3, pp. 150-157, 2015.

[5] Y. C. Pu and P. C. You, "Indoor positioning system based on BLE location fingerprinting with classification approach," Applied Mathematical Modelling, 2018.

[6] C. Zhou, J. Yuan, H. Liu, and J. Qiu, "Bluetooth Indoor Positioning Based on RSSI and Kalman Filter," Wireless Personal Communications, vol. 96, no. 3, pp. 4115-4130, 2017.

[7] Y. Gu, A. Lo, and I. Niemegeers, "A survey of indoor positioning systems for wireless personal networks," IEEE Communications Surveys and Tutorials, vol. 11, no. 1, pp. 13-32, 2009.

[8] W. Shao et al., "Location Fingerprint Extraction for Magnetic Field Magnitude Based Indoor Positioning," Journal of Sensors, vol. 2016, 2016.

[9] G. Kim and E. M. Petriu, "Fiducial marker indoor localization with Artificial Neural Network," IEEE/ASME International Conference on Advanced Intelligent Mechatronics, AIM, pp. 961-966, 2010.

[10] I. Igo Barandiaran, C. Paloc, and M. Grañ, "Real-time optical markerless tracking for augmented reality applications.'

[11] K.-W. Chen et al., "To Know Where We Are: Vision-Based Positioning in Outdoor Environments." 
[12] H. Subakti and J. R. Jiang, "A marker-based cyber-physical augmentedreality indoor guidance system for smart campuses," Proceedings - 18th IEEE International Conference on High Performance Computing and Communications, 14th IEEE International Conference on Smart City and 2nd IEEE International Conference on Data Science and Systems, HPCC/SmartCity/DSS 2016, pp. 1373-1379, 2017.

[13] B. Hartmann, N. Link, and G. F. Trommer, "Indoor 3D position estimation using low-cost inertial sensors and marker-based videotracking," Record - IEEE PLANS, Position Location and Navigation Symposium, pp. 319-326, 2010.

[14] J. P. Grisales Campeon, S. Lopez, S. R. De Jesus Melean, H. Moldovan, D. R. Parisi, and P. I. Fierens, "Fusion of magnetic and WiFi fingerprints for indoor positioning," in Congreso Argentino de Ciencias de la Informatica y Desarrollos de Investigacion, CACIDI 2018, 2018.

[15] E. Wang, M. Wang, Z. Meng, and X. Xu, "A Study of WiFi-Aided Magnetic Matching Indoor Positioning Algorithm," Journal of Computer and Communications, vol. 05, no. 03, pp. 91-101, 2017.

[16] K. Liu, G. Motta, T. Ma, and T. Guo, "Multi-floor Indoor Navigation with Geomagnetic Field Positioning and Ant Colony Optimization Algorithm," 2016.

[17] K. Liu, G. Motta, and T. Ma, "XYZ indoor navigation through augmented reality: A research in progress," Proceedings - 2016 IEEE
International Conference on Services Computing, SCC 2016, pp. 299306, 2016.

[18] H. Zhang, W. Lin, and A. Chen, "Path Planning for the Mobile Robot: A Review," Symmetry, vol. 10, no. 10, p. 450, Oct. 2018

[19] P. T. Mahida, S. Shahrestani, and H. Cheung, "Comparision of pathfinding algorithms for visually impaired people in IoT based smart buildings."

[20] K. Akaho, T. Nakagawa, Y. Yamaguchi, K. Kawai, H. Kato, and S. Nishida, "Route guidance by a car navigation system based on augmented reality," Electrical Engineering in Japan (English translation of Denki Gakkai Ronbunshi), vol. 180, no. 2, pp. 43-54, 2012.

[21] G. A. Lee, A. Dunser, A. Nassani, and M. Billinghurst, "AntarcticAR: An outdoor AR experience of a virtual tour to Antarctica," 2013 IEEE International Symposium on Mixed and Augmented Reality - Arts, Media, and Humanities, ISMAR-AMH 2013, no. Cci, pp. 29-38, 2013.

[22] M. J. Kim, X. Wang, S. Han, and Y. Wang, "Implementing an augmented reality-enabled wayfinding system through studying user experience and requirements in complex environments," 2015.

[23] "Positioning technology - IndoorAtlas." [Online]. Available: https://www.indooratlas.com/positioning-technology/. [Accessed: 28Nov-2019].

[24] "ARCore - Google Developers | Google Developers." [Online]. Available: https://developers.google.com/ar. [Accessed: 28-Nov-2019]. 